\title{
Research and Application of Moving Tracking of Stewart Based on Multi-innovation EKF Algorithm
}

\author{
Sujian Sheng ${ }^{1}$,Bo Yang ${ }^{1}$, Pinle Qin ${ }^{2}$, Xiaoqing Chen ${ }^{2}$ \\ 1 CNOOC Energy Technology \& Services- Oil Production Services Co. \\ 2 Department of Computer and Control Engineering, North University of China, Taiyuan 030051 \\ China \\ Pinle.qin@dlut.edu.cn
}

Keywords: Extend Kalman Filter; Multi-innovation; Multi-innovation Extend Kalman Filter;Simulation analyses

\begin{abstract}
Because of the low estimation accuracy of normal extended Kalman Filter in strong nonlinear system, an improved extended Kalman Filter (MI-EKF) is presented to solve the problem, and the filtering accuracy is greatly improved. In this paper, multi-innovation theory is applied to EKF, and the multi-innovation EKF (MI-EKF) is proposed. MI-EKF has better precision and stability, because MI-EKF considers not only the current measured value, but also give full consideration to the time before state of motion. Finally, the improvement algorithm is used the moving tracking of six degree freedom stewart motion platform, the simulation results show that the improved MI-EKF algorithm is superior to the standard EKFalgorithm.
\end{abstract}

\section{Introduction}

In the field of movement target tracking, Kalman Filter(KF) is suitable for realtime estimation in the case of a linear system, and it obtain the dynamic estimates of the target by minimum mean square error (MMSE)[1][2]. But strictly speaking, all the systems are dynamic and non-linear, and many of them are even strongly nonlinear system.Therefore, the movement target state estimation of nonlinear system possesses important theory significance and project application values.Extended Kalman filter (EKF)is one of the more widely used method for filtering, but it also has some deficiencies in the actual application. EKF is only suitable for weakly nonlinear systems, the performance of EKF is not stable in strongly nonlinear system, and there is also more chance of filter divergence situations occurring.Besides, if the second order or even higher order item of Taylor seriesin nonlinear observation equationcan't be ignored, the EKF filtering will be unstable. In order to improve the stability and the accuracy of EKF filtering, there are a lot of improved algorithms have been proposed. The authors of [5] come up with a strong tracking finite-difference Kalman filter (STFDEKF), in filtering calculation, strong tracking factor is introduced to modify priori covariance matrix to improve the accuracy of the filter. In [6], the authors put forward an improved EKF with multiple suboptimal fading factor (SMFEKF), which has a strong tracking filter performance, and the state estimation abilityof SMFEKF is also improved. In [7], an interpolation basedimprovedEKFalgorithm is presented, therecursion structure of EKFalgorithmis still adopted, and difference arithmetic based on Stirling's interpolation formula is used to substitute for derivativecalculation of nonlinear functions.Simulation results indicate thatinterpolation-based filteringcould obtainhigher stability andaccuracythan EKF. Because of the uncertain noise statistics in nonlinear system, a novel method is proposed in [8] and [9], they use the method of fictitious noisecompensating, and time-varying noise statistics call be estimated andcompensated on line, so itimproves the robustness of nonlinear filter.

Hence, based on the discussion above, in this paper, on the basis of standard EKF algorithm, withMulti-innovation identification theory, we put forward a new method, which is called Multi-innovation EKF (MI-EKF). The simulation results show that the improved MI-EKF algorithm is superior to the standard EKFalgorithm.

*Project supported by the youth fund of Shanxi Province (No.201002106-13). 


\section{Problem description and traditional extended Kalman filter(EKF)}

Consider the following nonlinear model in the rectangular coordinate system, the state equation and detecting equation are:

$$
\left\{\begin{array}{l}
x_{k}=f\left(x_{k-1}, \mathrm{u}_{k}, w_{k}\right) \\
z_{k}=h\left(x_{k}, v_{k}\right)
\end{array}\right.
$$

where $x_{k}$ is the matrix of the system state, $z_{k}$ is the observation matrix, $u_{k}$ is the inputof system, $f(\cdot)$ is the nonlinear mapping function, which can be used to calculate the predicted values from the data of previous state. Similarly, $h(\cdot)$ is can be used to calculatethe observed value of prediction by the predictive state, $w_{k}$ is the noise matrix of state, $v_{k}$ is noise matrix of measurement, $w_{k}$ and $v_{k}$ are independent of one another, and the mean and covariance of them are:

$$
\begin{aligned}
& E\left[w_{k}\right]=r, \operatorname{cov}\left[w_{k}, w_{j}\right]=R_{k} \delta(k-j), \\
& E\left[v_{k}\right]=q, \operatorname{cov}\left[v_{k}, v_{j}\right]=Q_{k} \delta(k-j)
\end{aligned}
$$

where, $\delta(k-j)$ is Kronecker delta.

In view of the nonlinear system (1), usually using EKF to realize the state estimate. But $f(\cdot)$ and $h(\cdot)$ cannot be applied in the covariancedirectly, Instead, the partial derivatives of them, that isJacobianmatrixes, are used in the covariance. In each step, the current estimated state is used to calculate the Jacobian matrix, in this way, the nonlinear function is linearized in its current state. In the EKF algorithm, state equation and observed equation of the original system are approximated with the Taylor series expansion. EKF algorithm is givenby the following recurrence formula:

Predictive equations:

$$
\begin{gathered}
\hat{x}_{k \mid k-1}=f\left(x_{k-1}, u_{k}, 0\right) \\
P_{k \mid k-1}=F_{k} P_{k-1 \mid k-1} F_{k}^{T}+Q_{k}
\end{gathered}
$$

Renewal equation:

$$
\begin{gathered}
\tilde{y}_{k}=z_{k}-h\left(\hat{x}_{k \mid k-1}, 0\right) \\
S_{k}=H_{k} P_{k \mid k-1} H_{k}^{T}+R_{k} \\
K_{k}=P_{k \mid k-1} H_{k}^{T} S_{k}^{-1} \\
\hat{x}_{k \mid k}=\hat{x}_{k \mid k-1}+K_{k} \tilde{y}_{k} \\
P_{k \mid k}=\left(I-K_{k} H_{k}\right) P_{k \mid k-1}
\end{gathered}
$$

where, $F_{k}=\left.\frac{\partial f}{\partial x}\right|_{\hat{x}_{k-1 \mid k-1}, u_{k}}, \quad H_{k}=\left.\frac{\partial h}{\partial x}\right|_{\hat{x}_{k \mid k-1}}, P_{k \mid k-1}$ is the covariance matrix of state $\hat{x}_{k \mid k-1}, P_{k \mid k}$ is the covariance matrix of state $\hat{x}_{k \mid k}, K_{k}$ is the Kalman gain. $\tilde{y}_{k}=z_{k}-h\left(\hat{x}_{k \mid k-1}, 0\right)$ isdefined asinnovation. Since EKF only use the first order item and ignore the higher order term when nonlinear function is linearized by the Taylor expansion, which only achieve partial linearization of nonlinear function, and it brings error to EKF when we use it for state estimation. As a result,the standard EKF has low accuracy, and may even lead to filter divergence. So it is very necessary to improve EKF for better filter performance. 


\section{Improvement of extended Kalman filter based on multi-innovation theory}

According to (5) and (8), only one innovation $\tilde{y}_{k}$ exists in the traditional EKF algorithm. The state prediction of time $k$ is only estimated by the status time $k-1$, and the past data information is underutilized, so that the useful information hidden in the past data is lost.Multi-innovation identification theory, proposed by Chinese scholar Ding Feng [11, 12], extends the scalar innovation to innovation vector, and the innovation vector to innovation matrix.As a result, he puts forward and sets up a kind of theory and method based on the idea of Multi-innovation, which is referred to asmulti-innovation identification theory and method, and includes the multi-step information in theiterative process [13].

\section{A. Multi-innovation identification theory}

Some identification algorithms [17], such as least squares [18] and stochastic gradient algorithm [19], have a common characteristic: all of them use a single innovation correction technology that uses single innovation correction technology.

For the following scalar systems [20]:

$$
y(k)=\varphi^{T}(k) \theta+v(k)
$$

where $y(k) \in \mathrm{R}$ is the output of the system, $\varphi^{T}(k)$ is the information vector that is formed by input-output data of the system, $\theta$ is the vector parameter to be identified, and $v(k)$ is the system noise.

To estimate the parameter vector $\theta$ in Formula (8)

$$
\hat{\theta}(k)=\hat{\theta}(\mathrm{k}-1)+L(k) e(k)
$$

where $L(k) \in R^{n}$ is the algorithm gain vector.(11) shows that, we can reformulate parameter estimation vector $\hat{\theta}(k)$ of time $k$ by the product of gain vector $L(k)$ and scalar innovation $e(k)$, which amends the estimated vector $\hat{\theta}(k-1)$ of time $k-1$. That means $\hat{\theta}(k)$ is calculated by adding the product of gain vector $L(k)$ and innovation $e(k)$ to $\hat{\theta}(k-1)$.

Innovation is an important quantity of recursion method, and it is used to describe output prediction error of time k.Innovation is defined as:

$$
e(k)=y(k)-\varphi^{T}(k) \hat{\theta}(\mathrm{k}-1) \in \mathrm{R}
$$

Innovation is different from the residuals, and the residuals are used to describe output deviation of time k.Residuals is defined as:

$$
\varepsilon(k)=y(k)-\varphi^{T}(k) \hat{\theta}(k) \in \mathrm{R}
$$

There is a relationship between theinnovation and the residuals:

$$
\varepsilon(k)=\frac{y(k)}{1+\Lambda(k) \varphi^{T}(k) P(k-1) \varphi(k)}
$$

or

$$
\varepsilon(k)=\left[1-\Lambda(k) \varphi^{T}(k) P(k) \varphi(k)\right] y(k)
$$

On the basis of single innovation, the scalar innovation $e(k) \in R$ is promoted as innovation vector

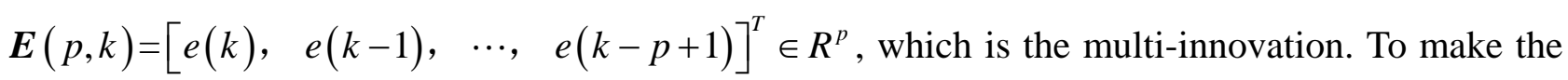
matrix multiplication dimension compatible, we extend gain vector $L(k) \in R^{n}$ to $\Gamma(p, k) \in R^{n \times p}$, then the multi-innovation identification algorithm turns out to be:

$$
\hat{\theta}(k)=\hat{\theta}(k-1)+\Gamma(p, k) \boldsymbol{E}(p, t)
$$

where $\Gamma(p, k) \in R^{n \times p}$ is the gain matrix, $E(p, k) \in R^{p}$ is the innovation vector, $p \geq 1$ is the Length 
of innovation. (10) shows that, in the multi-innovation identification algorithm, parameter estimation $\hat{\theta}(k)$ is corrected by the product of gain matrix $\Gamma(p, k)$ and innovation vector $\boldsymbol{E}(p, k)$, on the basis of parameter estimation $\hat{\theta}(k-1)$.

\section{B. Extended Kalman filterbased on multi-innovation(MI-EKF)}

Because the filter error of classical EKF will be enlarged or even lead to filterdivergence. To overcome the limitation, a novel method is proposed. Based on the abovemulti-innovation theory,we put forward a new method according to the standard EKF filtering divergence in the strongly nonlinear system,extend the originalsingleinnovation to multi-innovation. In (5) $e(k)=\tilde{y}_{k}=z_{k}-h\left(\hat{x}_{k \mid k-1}, 0\right)$ is defined as the innovation, and we extend it to innovation matrix:

$$
E(p, k)=\left[\begin{array}{c}
e(k) \\
e(k-1) \\
e(k-2) \\
\vdots \\
e(k-p+1)
\end{array}\right]=\left[\begin{array}{c}
z_{k}-h\left(\hat{x}_{k \mid k-1}, 0\right) \\
z_{k-1}-h\left(\hat{x}_{k-1 \mid k-2}, 0\right) \\
z_{k-2}-h\left(\hat{x}_{k-2 \mid k-3}, 0\right) \\
\vdots \\
z_{k-p+1}-h\left(\hat{x}_{k-p+1 \mid k-p}, 0\right)
\end{array}\right] \in R^{(m p)}
$$

By substituting (13) into (8), we can get the multi-innovation ExtendedKalman state prediction algorithm, and expand (8) to:

$$
\begin{aligned}
\hat{x}_{k \mid k} & =\hat{x}_{k \mid k-1}+\left[L_{1}(k), L_{2}(k), L_{3}(k) \cdots L_{p}(k)\right] E(p, k) \\
& =\hat{x}_{k \mid k-1}+\sum_{i=1}^{p} L_{i}(k) e(k-i+1)
\end{aligned}
$$

where, $\hat{x}_{k \mid k}$ is the current state of motion to be predicted, $\hat{x}_{k \mid k-1}$ is a state of motion before present instant, $L_{i}(k)(i=1,2 \cdots p)$ is the gain matrices of different time. $E(p, k)$ isinnovationmatrix, $p$ is the length ofinnovation.

When the MI-EKF is used to predict the state of motion, it not onlythinks about one state before present instant but it alsoconsiders fully other states even before.More motion information is contained in MI-EKF, and thefiltering accuracy of theimproved EKF issignificantly higher than that of EKF.We obtain the equations of MI-EKF as:

$$
\left\{\begin{array}{l}
\hat{x}_{k \mid k-1}=f\left(x_{k-1}, u_{k}, 0\right) \\
P_{k \mid k-1}=F_{k} P_{k-1 \mid k-1} F_{k}^{T}+Q_{k} \\
e(k-p+1)=z_{k-p+1}-h\left(\hat{x}_{k-p+1 \mid k-p}, 0\right),(p=1,2 \cdots) \\
S_{k}=H_{k} P_{k \mid k-1} H_{k}^{T}+R_{k} \\
K_{k}=P_{k \mid k-1} H_{k}^{T} S_{k}^{-1} \\
\hat{x}_{k \mid k}=\hat{x}_{k \mid k-1}+\sum_{i=1}^{p} L_{i}(k) e(k-i+1),(i=1,2 \cdots p) \\
P_{k \mid k}=\left(I-K_{k} H_{k}\right) P_{k \mid k-1}
\end{array}\right.
$$

In (19), we get the traditional EKF when $L_{1}(k)=L(k), L_{2}(k)=L_{3}(k)=L_{4}(k)=\cdots=L_{p}(k)=0$.

The improved MI-EKF proposed in this papercompared with the traditional EKF, MI- EKF has the following advantages: 
a) In each step of parameter estimation, standard EKF only uses the innovation at $k-1$ moment, but the improved MI-EKF not only uses the innovation at $k-1$ moment, but also uses the useful data and innovation in the past, so that the algorithm convergence is improved.

b) When predicted the state of time $k$, MI-EKF uses useful data of time k-1and timek-2. Similarly, when predicted the state of time $k+1$,MI-EKF uses useful data of time $k$ and time $k$ - 1 . Therefore, when predicted the state of two adjacent time (time $k$ and time $k+1$ ), it uses the useful data and innovation at $k-1$ moment repeatedly, and this is the main reasons for improving the MI-EKF algorithm accuracy.

\section{Experimental results}

In order to prove the effectiveness of the proposed MI-EKF, We selected six degree of freedom motion platform control system as the research object, as shown in the Fig. 1. and analysis the moving tracking effect. EKF and MI-EKF are used respectively to predict the system state in the motion platform that is nonlinear systems. In the experimental process, sine signal is generated, it highlights the advantages of MI-EKF by comparing filter performance of the two filters, at the same time, the root of mean square error (RMSE) is used to make the quantitative analysis of filter performance.In the traditional EKF algorithm, there is only one innovation, the improved MI-EKF in this paper addsanother oneinnovation, that is to say, the state prediction of time $k$ is estimated by the status time $k$-1and time $k-2$.

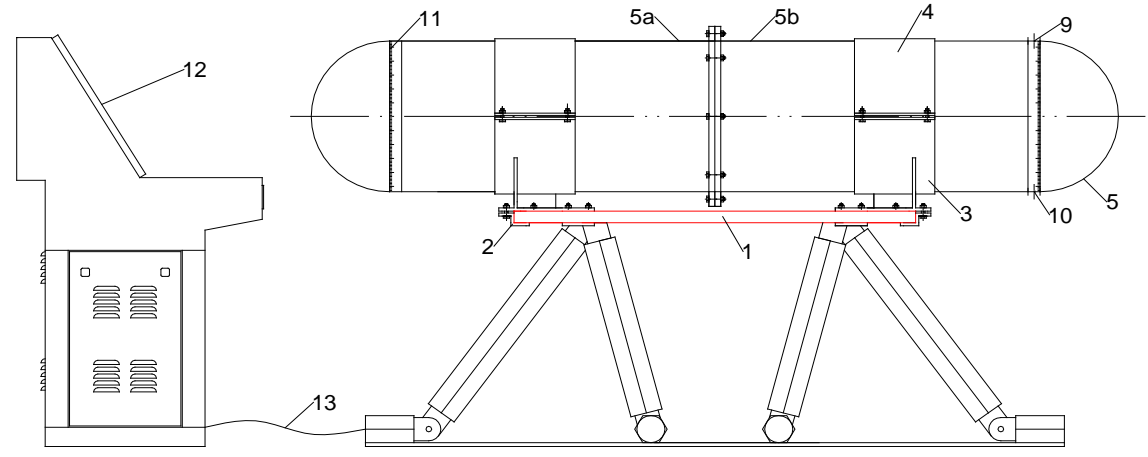

Fig.1 The system principle diagram

Use the following nonlinear system model: The sine signal of system could be expressed as following:

$$
\begin{gathered}
\sin \left(x_{k}\right)=f\left(x_{k-1}, \mathrm{u}_{k}, w_{k}\right) \\
=F \sin \left(x_{k-1}\right)+w_{k}
\end{gathered}
$$

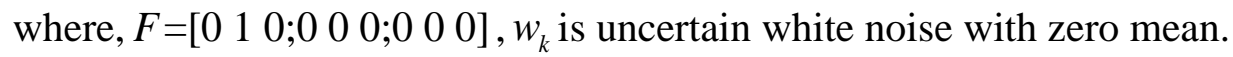

The initialization of filter (both EKF and MI-EKF have the same initialization)as follows:

The angle of sin function: $v=0$

The angular velocity of sin function: $w=10$

The amplitude of sin function: $a=1$

Set the initial vector: $M=[v, w, a]$

The sample time is $6 \mathrm{~s}$, sampling numbers are 500 , and the sampling interval is $6 / 500 \mathrm{~s}$. In fig.2, we can see the true value and the observed value. 


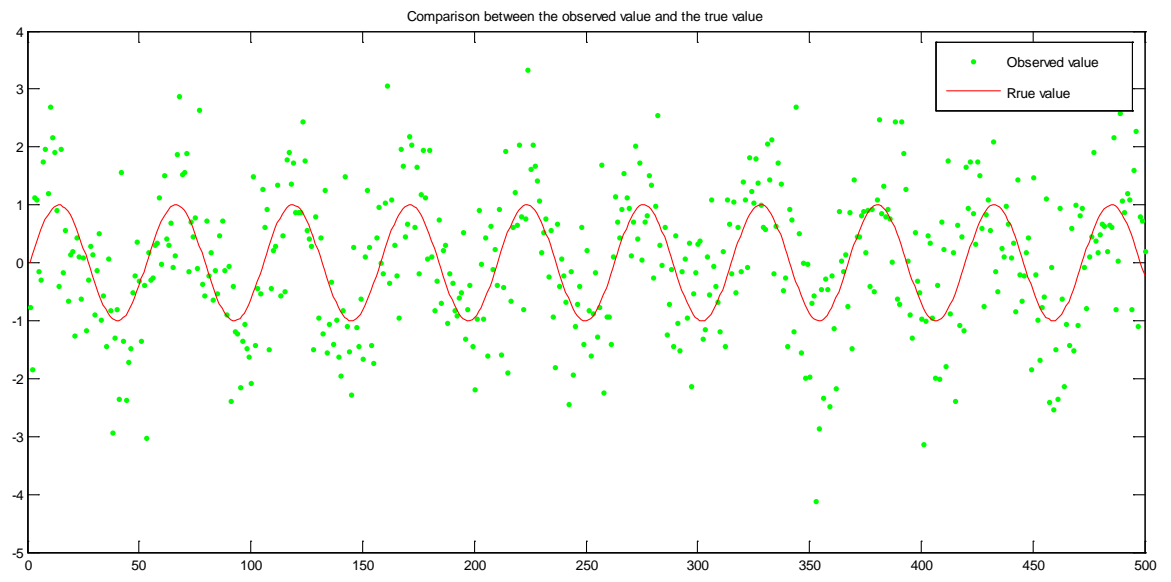

Fig.2: the true value and the observed value

The filter performances are showed in Fig.3. In Fig.3, the redline represents the actual observation data ofnonlinear system, and the blue line represents the predicted trajectory of standard EKFalgorithm, and the black linerepresents the predicted trajectory of the improved MI-EKF forecasting algorithm. Figure 3 shows that there is large error between the filtering trajectory and the actual trajectory although the standard EKF algorithm can meet the requirements of the filtering, and the filtering effect is not ideal when the filtering precision is higher, but the improved MI-EKF forecasting algorithm has higher prediction accuracy.

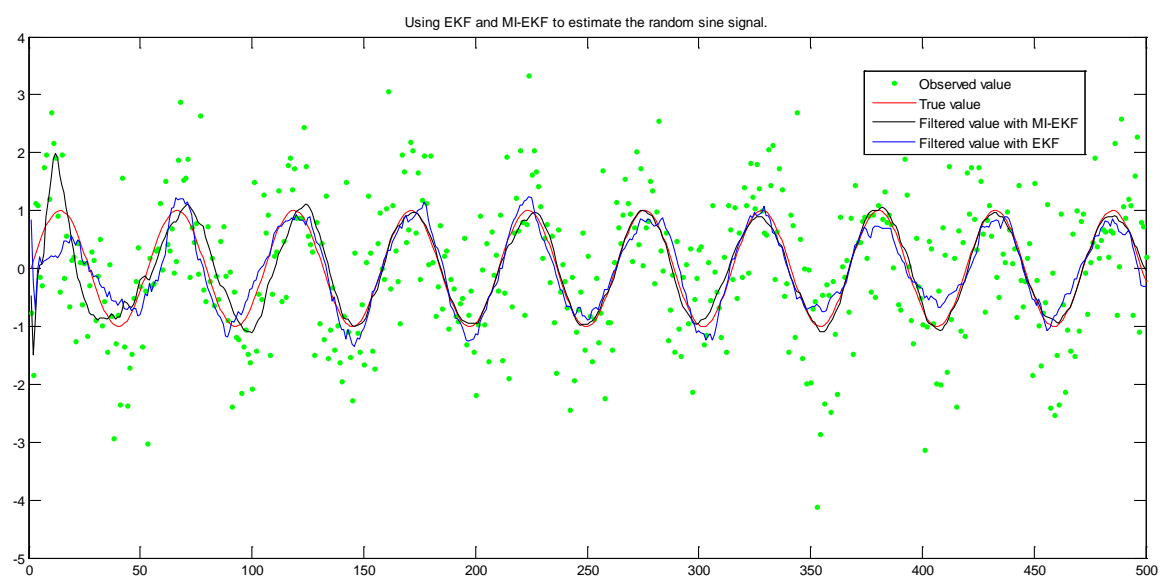

Fig.3:state estimation by EKF and MI-EKF

Fig.4 shows the estimated error of EKF and MI-EKF. The blue line represents the filtering errors of standard EKF algorithm, and the black linerepresents the filtering errorsof the improved MI-EKF forecasting algorithm.It can be seen from figures that the improved MI-EKF algorithm is significantly better than standard EKF, and the accuracy of improved MI-EKF algorithm forecast result is higher. 


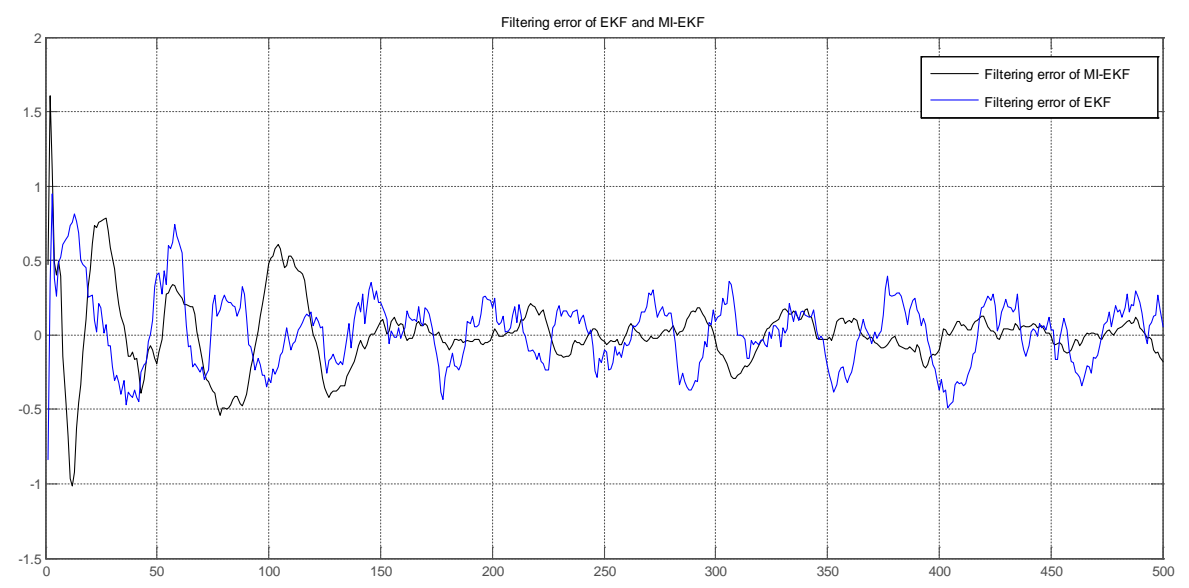

Fig.4: The state estimated error by EKF and MI-EKF

\section{Conclusions}

In allusion to thedisadvantage thatthe standard extended Kalman filterhas low filtering precision, an improved algorithm based on multi-innovation identification theory (MI-EKF) is proposed in this paper.This algorithm gives full consideration to the useful information before the current time, and the improved MI-EKF algorithm is relatively easy to understand and implement. Simulation experiment and theoretical analysis showthat, comparedwith EKF, the improved MI-EKFalgorithmobtainshigher stability and accuracythan standardEKFalgorithm, and has better performance and larger application area.

\section{Acknowledgement}

This work is partially supported by Shanxi Youth Foundation (No.2010021016-3). The authors also gratefully acknowledge the helpful comments and suggestions of the reviewers, which have improved the presentation.

\section{References}

[1] Zhang Rui, Shi Linan.Tracking of maneuvering target basedon extendedKalmanFilter[J].AerospaceControl,2012,30(3):12-17.

[2] O. A. Stepanov. Kalman filtering: Past and present. An outlook from Russia. (On the occasion of the 80th birthday of Rudolf Emil Kalman) [J]. Gyroscopy and Navigation.2011, 2(2):99-110.

[3] Yang Hong, LiYaan, LI Guohui. New method of improved extended Kalman filter[J]. Computer Engineering and Applications, 2010,46(19):18-20.

[4] Quan-bo Ge, Wen-bin Li, Cheng-lin Wen. SCKF-STF-CN: a universal nonlinear filter for maneuver target tracking [J]. Journal of Zhejiang University SCIENCE C, 2011, 12(8):678-686.

[5] Fan Wenbing, Liu Chunfeng, ZhangSuzhen.Improved method of strong tracking extended Kalman filter. Control and Decision, 2006, 20(1):73-76.

[6] Zhou Donghua, Xi YuGen, ZhangZhongjun.A multiple suboptimal fading factor of extended Kalman filter.Actaautomaticasinica, 1991, 17(6):689-758.

[7] Liu Guohai, Shi Wei, Li Kangji.Applicationofinterpolation-based improvedEKFalgorithminintegrated navigation. Chinese Journal of Scientific Instrument,2007,28(10):1897-1901.

[8] Arie Berman, Joshua Dayan. Improved EKF Method of Estimating Locations with Sudden High Jumps in the Measurement Noise. Journal of Intelligent and Robotic Systems, 2001, 32(3): 461-476. 
[9] WuZhihua,Ding Yangbin, ShenGongxu.Modified nonlinear robust EKF algorithm and its applications. ComputerEngineeringandApplications, 2011, 47(3):207-209.

[10]Li Li.Fading memory extended Kalman filtering algorithmfor maneuvering target tracking application[J]. Electronic measurement technology, 2011,34(2):36-38.

[11]Ding F, Xiao D and Ding Tao. Multi-innovation stochastic gradient identification methods. Control Theory and Application.2003, 20(6):870-874.

[12]Ding F. Several multi-innovation identification methods. Digital Signal Processing, 2010, 20(4):1027-1039.

[13]Ding J, Xie L, Ding F. Performance analysis of multi-innovation stochastic gradient identification for non-uniformly sampled systems. Control and Decision, 2011, 26(9):1338-1342.

[14]Ding F. System identification - Part A: Introduction to the identification. Journal of Nanjing University of Information Science \& Technology (Natural Science Edition), 2011, 3(1):1-22.

[15]Liu YJ, Ding F, Hierarchical least squares identification method for periodically non-uniformly sampled systems. Control and Decision, 2011, 26(3):453-456.

[16]Ding J, Xie L, Ding F. Performance analysis of multi-innovation stochastic gradient identification for non-uniformly sampled systems. Control and Decision, 2011, 26(9):1338-1342.

[17]Ding F. System identification. Part F: Multi-innovation identification theory and methods, Journal of Nanjing University of Information Science \& Technology, 2012, 4(1):1-28. 